\section{Tetrahedral Co-ordination of Nickel(II)}

IN a recent communication ${ }^{1}$, Katzin has interpreted the appearance of blue colour in paramagnetic nickel(II) compounds as indicative of tetrahedral co-ordination. We have recently completed the determination of the crystal structure of $\mathrm{Ni}(e n)_{2}$ (NCS) ${ }_{2}$ (Brown, B. W., and Lingafelter, E. C., unpublished work). This compound is blue and paramagnetic, but the co-ordination of the nickel ion is transoctahedral, with $\mathrm{Ni}-\mathrm{N}$ distances of $2 \cdot 10 \mathrm{~A}$. to the ethylenediamine and $2 \cdot 15 \mathrm{~A}$. to the isothiocyanate. We are also investigating the crystal structure of $\mathrm{Ni}(\mathrm{en})\left(\mathrm{H}_{2} \mathrm{O}\right)_{4}\left(\mathrm{NO}_{3}\right)_{2}$, another blue paramagnetic compound. Although the study is still in the early stages and all atoms have not been located, the presence of a co-ordination octahedron about the nickel atom is already clear.

Thus these two compounds show that the blue colour cannot be taken as an indication of tetrahedral co-ordination of nickel(II).

Department of Chemistry,

University of Washington, Seattle 5, Washington. Nov. 10.

1 Katzin, L. I., Nature, 182, 1013 (1958).

\section{A Method for improving the Solute} Selectivity of Osmotic Membranes

As examination of the results obtained with a pair of 'Ultracella' filters (fein)-manufactured by the Membranfiltergesellschaft, Göttingen-which had been used for several months in benzene at $30^{\circ} \mathrm{C}$. to determine the number-average molecular weights $\left(\bar{M}_{n}\right)$ of polymer samples, showed that during this time the membranes had become markedly less permeable to solvent. A similar observation has been made by Philipp and Bjork' with a gel 'Cellophane' membrane, and they attributed the phenomenon to gradual ageing of the membranes. However, with the 'Ultracella' filters (fein) closer examination of the results indicated that the change in permeability had occurred in two distinct stages both of which could be associated with attempts to measure the $\bar{M}_{n}$ of a sample of poly(diallyl phthalate). Experiments were therefore carried out with three other pairs of 'Ultracella' filters (fein) in order to investigate this phenomenon further.

The membranes were assembled in standard ZimmMyerson type osmometers and before and after treat. ment with solutions of poly(diallyl phthalate) were characterized by $(a)$ the solvent permeability, measured by the rate of fall of the meniscus in the measuring capillary under a head of $10 \mathrm{~cm}$. of benzene, and (b) the solute selectivity, measured by the maximum osmotic head developed with a 1 per cent ( $w / w$ ) benzene solution of a commercial polystyrene which has a very wide distribution of molecular weights 2 .

The results in Table 1 show that in each case after treatment with poly(diallyl phthalate) the mem. branes became less permeable and more selective (that is, gave higher osmotic heads). Furthermore, these experiments show that the magnitudes of these effects $(a)$ increased with poly(diallyl phthalate) concentration (membranes UCF/F 457/A1 and UCF/F 1256/4) up to a maximum concentration of about 1.5 per cent ( $w / v$ ) (membranes UCF/F 1256/5), (b) after $16 \mathrm{hr}$. were independent of the time the membranes were in contact with the poly(diallyl phthalate) solution (membranes UCF/F 1256/4), $(c)$ in the molecular weight-range $13,000-22,000$ wer $\theta$

Table 1. Mrasurements in Benzene at $30^{\circ} \mathrm{O}$.; 'Ulmrachlla' Filmers (Fhis) (UCF/F)

\begin{tabular}{|c|c|c|c|c|c|c|c|}
\hline \multicolumn{4}{|c|}{ Treatment } & \multirow{2}{*}{\multicolumn{2}{|c|}{$\frac{\text { Permeability }}{\begin{array}{c}\text { Fall from } 10 \mathrm{~cm} . \\
\text { head of benzene }\end{array}}$}} & \multirow{2}{*}{\multicolumn{2}{|c|}{$\frac{\text { Selectivity }}{\begin{array}{c}\text { Equilibrium osmotic pressure } \\
\text { with } 1 \text { per cent }(w / w) \text { commercial } \\
\text { polystyrene }\end{array}}$}} \\
\hline \multicolumn{3}{|c|}{ Poly(diallyl phthalate) solution } & \multirow{2}{*}{$\begin{array}{l}\text { Time left in } \\
\text { osmometer } \\
\text { (hr.) }\end{array}$} & & & & \\
\hline Sample & $\overline{M_{n}}$ & $\begin{array}{l}\text { Concentration } \\
\text { (per cent) } \\
\text { (w/v) }\end{array}$ & & 5 min. & $\begin{array}{l}30 \mathrm{~min} . \\
(\mathrm{cm} .)\end{array}$ & $\begin{array}{c}\text { Pressure } \\
\text { (cm.) }\end{array}$ & $\begin{array}{l}\text { Time } \\
\text { (hr.) }\end{array}$ \\
\hline \multicolumn{8}{|c|}{$\mathrm{UCF} / \mathrm{F} 457 / A 1$} \\
\hline $\begin{array}{l}10 \cdot 1 \\
10 \cdot 1\end{array}$ & $\begin{array}{l}13,000 \\
13,000\end{array}$ & $\begin{array}{l}0 \cdot 1 \\
1 \cdot 5\end{array}$ & $\begin{array}{l}16 \\
16\end{array}$ & $\begin{array}{l}1 \cdot 31 \\
0 \cdot 74 \\
0 \cdot 31\end{array}$ & $\begin{array}{l}5 \cdot 85 \\
3 \cdot 86 \\
1 \cdot 77\end{array}$ & $\begin{array}{l}3 \cdot 35 \\
4 \cdot 93 \\
6 \cdot 01\end{array}$ & $\begin{array}{r}<16 \\
6 \\
6\end{array}$ \\
\hline \multicolumn{8}{|c|}{$\mathrm{UCF} / \mathrm{F} 1256 / 4$} \\
\hline $\begin{array}{l}\overline{B S I} \\
B S I \\
B S I\end{array}$ & $\begin{array}{l}22,000 \\
22,000 \\
22,000\end{array}$ & $\begin{array}{l}-\overline{0 \cdot 1} \\
0 \cdot 1 \\
1 \cdot 5\end{array}$ & $\begin{array}{l}\overline{16} \\
64 \\
16\end{array}$ & $\begin{array}{l}1 \cdot 25 \\
0 \cdot 92 \\
0 \cdot 68 \\
0 \cdot 33\end{array}$ & $\begin{array}{l}5 \cdot 04 \\
4 \cdot 03 \\
3 \cdot 35 \\
1 \cdot 91\end{array}$ & $\begin{array}{l}4 \cdot 43 \\
4 \cdot 65 \\
4 \cdot 97 \\
5 \cdot 94\end{array}$ & $\begin{array}{r}4 \\
4 \frac{41}{2} \\
<18 \\
<21 \frac{1}{2}\end{array}$ \\
\hline \multicolumn{8}{|c|}{ UCF/F $1256 / 5$} \\
\hline $\begin{array}{l}\overrightarrow{B S I} \\
10 \cdot 1\end{array}$ & $\begin{array}{l}2 \overline{2,000} \\
13,000\end{array}$ & $\overline{1 \cdot 5}$ & $\begin{array}{l}\overline{64} \\
16\end{array}$ & $\begin{array}{l}1 \cdot 58 \\
0 \cdot 46 \\
0 \cdot 51\end{array}$ & $\begin{array}{l}6 \cdot 27 \\
2 \cdot 56 \\
2 \cdot 62\end{array}$ & $\begin{array}{l}3 \cdot 93 \\
5 \cdot 83 \\
5 \cdot 72\end{array}$ & $\begin{array}{c}3 \\
<2 \frac{1}{2} \\
8\end{array}$ \\
\hline
\end{tabular}

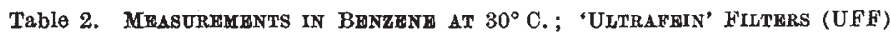

\begin{tabular}{|c|c|c|c|c|c|c|c|}
\hline \multicolumn{4}{|c|}{ Treatment } & \multicolumn{2}{|c|}{ Permeability } & \multicolumn{2}{|c|}{ Selectivity } \\
\hline \multicolumn{3}{|c|}{ Poly(diallyl phthalate) solution } & \multirow[b]{2}{*}{$\begin{array}{l}\text { Time left in } \\
\text { osmometer } \\
\text { (hr.) }\end{array}$} & \multicolumn{2}{|c|}{$\begin{array}{l}\text { Fall from } 10 \mathrm{~cm} \text {. head of } \\
\text { benzene }\end{array}$} & \multicolumn{2}{|c|}{$\begin{array}{l}\text { Equilibrium osmotic pressure } \\
\text { with } 1 \text { per cent }(w / w) \text { commercial } \\
\text { polystyrene }\end{array}$} \\
\hline Sample & $\widetilde{M}_{n}$ & $\begin{array}{l}\text { Concentration } \\
(\text { per cent) } \\
(w / v)\end{array}$ & & 5 min. & $30 \mathrm{~min}$. & $\begin{array}{c}\text { Pressure } \\
\text { (cm.) }\end{array}$ & Time \\
\hline \multicolumn{8}{|c|}{ 'Ultrafein' filter (gross) (UFF/G 1157/1) } \\
\hline$\overline{B S I}$ & 22,000 & $\overline{1 \cdot 5}$ & $\overline{66}$ & $\begin{array}{l}2 \cdot 96 \\
0 \cdot 74\end{array}$ & $=$ & $\begin{array}{l}5 \cdot 83 \\
7 \cdot 99\end{array}$ & $\sim 20^{2 \frac{1}{2}}$ \\
\hline \multicolumn{8}{|c|}{ 'Ultrafein' fllter (fein) (UFF/F 457/2) } \\
\hline $\overrightarrow{B S T}$ & -700 & $\overline{1.5}$ & $-\overline{18}$ & $1 \cdot 20$ & $4 \cdot 89$ & $4 \cdot 94$ & 22 \\
\hline \multirow{2}{*}{\multicolumn{8}{|c|}{ 'Ultrafein' fliter (allerfeinst) (UFF/AF' 457/A3) }} \\
\hline & & & & 0.045 & $0 \cdot 296$ & & \\
\hline $2 B$ & 25,400 & 0.4 & 352 & 0.050 & $0 \cdot 305$ & $8 \cdot 31$ & 166 \\
\hline
\end{tabular}

